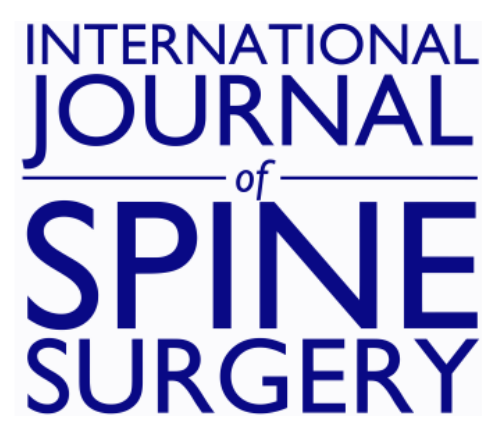

\title{
Editors' Introduction: Biologics and Regenerative Medicine in Intervertebral Disc Regeneration and Spinal Fusion
}

Roger Härtl, Jacobus J.C. Arts and Hyun W. Bae

Int J Spine Surg 2021, 15 (s1) 8-9

doi: https://doi.org/10.14444/8051

http://ijssurgery.com/content/15/s1/8

This information is current as of April 26, 2023.

Email Alerts Receive free email-alerts when new articles cite this article. Sign up at:

http://ijssurgery.com/alerts

The International Journal of Spine Surgery

2397 Waterbury Circle, Suite 1,

Aurora, IL 60504, Phone: +1-630-375-1432 


\title{
Editors' Introduction: Biologics and Regenerative Medicine in Intervertebral Disc Regeneration and Spinal Fusion
}

\author{
ROGER HÄRTL, MD ${ }^{1}$ JACOBUS J.C. ARTS, PHD,${ }^{2,3}$ HYUN W. BAE, MD ${ }^{4}$ \\ ${ }^{1}$ Weill Cornell Center for Comprehensive Spine Care, New York, New York ${ }^{2}$ Department of Orthopaedic Surgery, Maastricht University Medical Centre, \\ Maastricht, the Netherlands ${ }^{3}$ Department of Orthopaedic Biomechanics, Faculty of Biomedical Engineering, Eindhoven University of Technology, Eindhoven, \\ the Netherlands ${ }^{4}$ The Spine Institute Center for Spinal Restoration, Santa Monica, California
}

Keywords: biologics, regenerative medicine

Degenerative disc disease (DDD) affects the intervertebral discs (IVDs) that separate the vertebral bodies in the spinal column.

Age-related and pathology-driven changes in spinal physiology and anatomy can lead to arthritis, disc herniation, or spinal stenosis. Management of spine pathology frequently leads to surgical intervention in the form of spinal fusion, where bone growth between endplates of consecutive spinal vertebrae is required to permanently connect two or more vertebrae to improve spinal stability, correct a spinal deformity, or reduce pain. Currently, there is an abundance of available (bio)materials and surgical techniques in the field of spinal fusion surgery, and much research effort is geared toward achieving regeneration of IVDs through numerous approaches.

The first section of this special issue summarizes the literature and current status regarding IVD degeneration research and treatment options.

Kirnaz et al focus on the pathomechanism and biomechanics of DDD and how impaired cell metabolism due to $\mathrm{pH}$ alteration and poor nutrition may lead to autophagy and disruption of homeostasis within the IVD, thus playing a key role in DDD etiology. To develop regenerative therapies for degenerated discs, future studies must aim to restore both anatomical and biomechanical properties of the IVDs. The objective of their review is to provide a detailed overview of anatomical, radiological, and biomechanical features of IVDs as well as discuss the structural and functional changes during the degeneration process.

Jerome et al focus on the risks of intradiscal injections with orthobiologics and provide a review of literature and case series with a focus on the use of mesenchymal signaling cells and platelet-rich plasma (PRP) preparations. Although biologic intradiscal injection shows promise for the treatment of discogenic back pain, there are inherent risks to be considered and mitigated. The choice of injectable orthobiologics may matter, and in the authors' clinical experience, the risk appears higher with bone marrow concentrate than with PRP.

Vadalà et al describe the use of stem cells in IVD regeneration and provide the rationale behind a regenerative stem cell therapy for IVD degeneration. They also describe the effect of stem cells following their implantation in the disc environment. Furthermore, they present clinical evidence while taking into account the future perspectives and current limitations of this cutting-edge therapy.

Singh et al describe the role and evidence for non-stem cell-based regenerative therapies for IVD degeneration. They conclude that non-stem cellbased therapies, which include growth factor therapy and other proteins, can lead to an increased production of collagen and proteoglycans within the disc, potentially starting regeneration of the IVD.

The second part of this special issue is focused on use of biomaterial scaffolds for use in spinal fusion surgery. A current status of the literature is provided along with lessons learned. Considering the constant motion of new materials for spinal fusion, these updates are much needed and lay the foundation for a future update on the "ISASS Recommendations and Coverage Criteria for Bone Graft Substitutes Used in Spinal Surgery."1

Although autograft is still considered the "gold standard" in spinal fusion, there are drawbacks to its use, such as its limited availability and donor-site morbidity. In the past decades, these drawbacks 
have generated a vast interest in the development of new bone graft materials, particularly of ceramic origin but also stem cells, blood-derived biomaterials, and new demineralized bone matrix (DBM) formulations, all of which are available on the market. With the arrival of these new biomaterial formulations, we are entering the era of biomimetic materials; however, despite the high interest, indepth knowledge about their function and clinical performance is still lacking.

To this extent, Cohen et al focus on allografts. The manuscript provides an overview on the different subtypes with an emphasis on recent product development of allografts and its use in spinal fusion surgery. The authors conclude that there is great need for improvement in allografts.

The comprehensive literature review from Stephan et al focuses on preclinical and clinical studies detailing the application of mesenchymal stem cells (MSCs) in spinal fusion surgery. Although MSCs may be a promising option to use to augment grafting for spinal fusion surgery, they must be used with appropriate osteoconductive scaffolds. Additional studies are necessary to determine the efficacy of MSCs with different osteoconductive scaffolds and growth/osteogenic differentiation factors.

Plantz et al review current evidence and future trends of various classes of synthetic bone graft materials in spine fusion. In their conclusion, they state that the ability to finely tune the biologic properties of various synthetic materials has spurred significant growth in the field of bone grafts. Synthetics - including ceramics, glass ceramics, and polymer-based compounds - continue to yield excellent efficacy as bone graft extenders across a variety of spinal fusion procedures while minimizing the need for autograft. While many synthetics, such as ceramics, have historically been used in combination with autograft as bone graft extenders, current translational research suggests that perhaps synthetic and biologic composites could yield adequate bone healing without the need for autograft or high-dose growth factors.

Shepard et al provide an insightful review of current applications and future trends of DBM in spine surgery. Although DBM has been successfully used as bone graft extenders in spinal surgery yielding good fusion rates, care should be taken when using as a stand-alone product. As new literature emerges, DBM and demineralized bone fibers can be useful methods in a surgeon's armamentarium for fusion-based procedures. As a future trend, the combination of additional cellbased products with demineralized bone fibers could be an exciting opportunity in spine surgery.

Together, the review articles and fundamental studies in this special issue provide the reader with an excellent overview of the current state of IVD regeneration approaches and on the use of bone grafts and biomaterials in spinal fusion. This special issue is by no means intended as a comprehensive overview, but it aims to raise awareness and stimulate discussion regarding regeneration of the IVD and the use of bone grafts and biomaterials in spinal fusion. We hope that this special collection of papers will provide you with the type of information that can contribute to your practice in terms of both treatment decisions and patient counseling and that you will find this a useful addition to your clinical practice and education.

\section{REFERENCES}

1. Abjornson C, Brecevich A, Callanan T, Dowe C, Camissa FP, Lorio MP. ISASS recommendations and coverage criteria for bone graft substitutes used in spinal surgery. Int $J$ Spine Surg. 2018;12(6):757-771. doi:10.14444/5095

Disclosures and COI: Dr Härtl is a consultant for Ulrich, Brainlab, and DePuy-Synthes, and he has royalties from Zimmer. Dr Arts is a consultant for Bonalive, Cerapedics, and DSM Biomedical. Dr Bae has no conflicts to disclose.

Corresponding Author: Roger Härtl, MD, Professor of Neurological Surgery, Director of Spinal Surgery, Director of the Weill Cornell Center for Comprehensive Spine Care, Department of Neurological Surgery, Starr Building, Room 651, 525 East 68th Street, Box 99, New York, NY 10021. Phone: (212) 746-2152; Email: roh9005@med. cornell.edu.

Published 30 April 2021

This manuscript is generously published free of charge by ISASS, the International Society for the Advancement of Spine Surgery. Copyright (c) 2021 ISASS. To see more or order reprints or permissions, see http://ijssurgery.com. 